University of Nebraska - Lincoln

DigitalCommons@University of Nebraska - Lincoln

2010

\title{
Interlayer exchange coupling across a ferroelectric barrier
}

M Ye Zhuravlev

University of Nebraska-Lincoln, myezhur@gmail.com

A V. Vedyayev

Moscow State University

Evgeny Y. Tsymbal

University of Nebraska-Lincoln, tsymbal@unl.edu

Follow this and additional works at: https://digitalcommons.unl.edu/physicstsymbal

Part of the Condensed Matter Physics Commons

Zhuravlev, M Ye; Vedyayev, A V.; and Tsymbal, Evgeny Y., "Interlayer exchange coupling across a ferroelectric barrier" (2010). Evgeny Tsymbal Publications. 37.

https://digitalcommons.unl.edu/physicstsymbal/37

This Article is brought to you for free and open access by the Research Papers in Physics and Astronomy at DigitalCommons@University of Nebraska - Lincoln. It has been accepted for inclusion in Evgeny Tsymbal Publications by an authorized administrator of DigitalCommons@University of Nebraska - Lincoln. 


\title{
FAST TRACK COMMUNICATION
}

\section{Interlayer exchange coupling across a ferroelectric barrier}

\author{
M Ye Zhuravlev ${ }^{1,2}$, A V Vedyayev $^{3}$ and E Y Tsymbal ${ }^{1}$ \\ ${ }^{1}$ Department of Physics and Astronomy, Nebraska Center for Materials and Nanoscience, \\ University of Nebraska, Lincoln, NE 68588, USA \\ ${ }^{2}$ Kurnakov Institute for General and Inorganic Chemistry, Russian Academy of Sciences, \\ Moscow 119991, Russia \\ ${ }^{3}$ Department of Physics, M V Lomonosov Moscow State University, Moscow 119899, Russia \\ E-mail: myezhur@gmail.com and tsymbal@unl.edu
}

Received 12 July 2010

Published 2 August 2010

Online at stacks.iop.org/JPhysCM/22/352203

\begin{abstract}
A new magnetoelectric effect is predicted originating from the interlayer exchange coupling between two ferromagnetic layers separated by an ultrathin ferroelectric barrier. It is demonstrated that ferroelectric polarization switching driven by an external electric field leads to a sizable change in the interlayer exchange coupling. The effect occurs in asymmetric ferromagnet/ferroelectric/ferromagnet junctions due to a change in the electrostatic potential profile across the junction affecting the interlayer coupling. The predicted phenomenon indicates the possibility of switching the magnetic configuration by reversing the polarization of the ferroelectric barrier layer.
\end{abstract}

(Some figures in this article are in colour only in the electronic version)

Materials and structures with coupled magnetic and electric characteristics have recently attracted significant interest due to intriguing physical properties and potential applications [1-6]. The coupling occurs through a magnetoelectric effect, which is considered to be a prospective way to manipulate the material's magnetization by applying an electric field. Bulk multiferroics are among the most familiar materials of this type [1]. Other structures which demonstrate coupled electric and magnetic order parameters are heterostructures composed of dielectric (ferroelectric) and ferromagnetic components. In these heterostructures the magnetoelectric coupling occurs across interfaces and may be much stronger than in bulk multiferroics [2-5]. In a broader vision magnetoelectric effects also involve electrically controlled interface magnetic order [7, 8], exchange bias [9-12], spin transport [13-17], and magnetocrystalline anisotropy [18-24].

Several known mechanisms give rise to magnetoelectric effects. Intrinsic magnetoelectric coupling occurs in compounds with no time-reversal and no space-inversion symmetries [1] where an external electric field displaces the magnetic ions, altering the magnetic properties of the compound [25].
Magnetoelectric coupling may also occur in composites of electrostrictive and magnetostrictive compounds where the order parameters are affected by mechanical strain coupling between the constituents [26, 27]. At ferromagnet/insulator interfaces the magnetoelectric effect may originate from purely electronic mechanisms. It was predicted that atomic displacements at the ferromagnet/ferroelectric interface caused by ferroelectric switching change the overlap between atomic orbitals at the interface, which in turn affects the interface magnetization [28, 29]. Recently it was demonstrated that a magnetoelectric effect can be induced by free carriers [30]. In this case, due to spin-dependent screening [31], an applied electric field produces an accumulation of spin-polarized electrons or holes at the metal-insulator interface resulting in a change of the interface magnetization [18] and exchange splitting [32, 33].

In this paper we predict a new magnetoelectric effect originating from the interlayer exchange coupling (IEC) between two ferromagnetic layers separated by an ultrathin ferroelectric barrier. It is known that magnetizations of two ferromagnetic layers separated by a non-magnetic metal or thin 


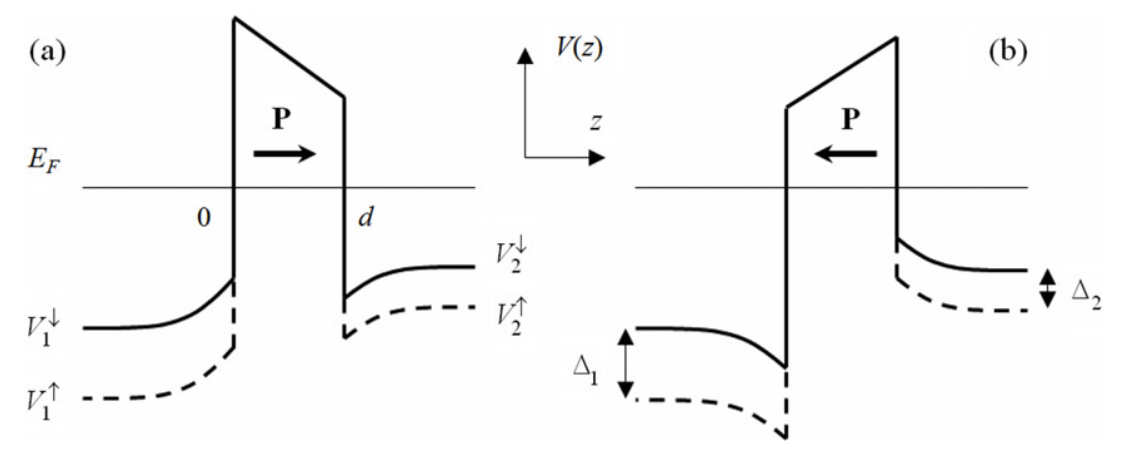

Figure 1. Schematic representation of the potential profile across the junction for two opposite directions of ferroelectric polarization.

dielectric barrier are exchange coupled $[34,35]$. In the case of a metallic spacer, the IEC is controlled by the RudermanKittel-Kasuya-Yoshida (RKKY) interaction that is mediated by the conduction electrons of the metal spacer layer and results in IEC oscillations with spacer thickness [35]. In the case of an insulating barrier, the IEC is determined by evanescent states in the barrier and exponentially decreases with barrier thickness. The IEC is interrelated with the relevant phenomenon of tunneling magnetoresistance (TMR) which is observed in magnetic tunnel junctions [36, 37]. In some cases both the IEC and TMR may be assisted by resonant effects [38, 39]. Experimental observation of the IEC across a tunnel barrier is more demanding compared to metallic spacers because of the rapid decay of the IEC amplitude with barrier thickness. Nonetheless, measurements of the IEC across a $\mathrm{MgO}$ barrier have been performed and showed a sizable magnitude [40, 41].

Use of a nanometer-thick ferroelectric barrier as a spacer layer allows us to control the magnitude (and sign) of the IEC by an external electric field through ferroelectric polarization switching. The effect occurs due to a change in the electrostatic potential profile across the junction with asymmetric interfaces similar to that in ferroelectric [42] and multiferroic tunnel junctions [43]. The change in the electrostatic potential affects the evanescent states and changes the electronic properties of the interfaces responsible for the IEC. Thus, switching the ferroelectric polarization of an ultrathin ferroelectric barrier makes it possible to affect the IEC and hence allows control of magnetic properties by electric means.

To calculate the IEC we consider two semi-infinite ferromagnetic electrodes separated by a thin ferroelectric barrier of thickness $d$. We use a free-electron model within an effective mass approximation to describe the electronic structure of the system. The exchange splittings of the freeelectron bands in the left and right electrodes are introduced via parameters $\Delta_{n}$ such that the spin-dependent potentials in the ferromagnetic layers are given by $V_{n}^{\sigma}=V_{n} \pm 1 / 2 \Delta_{n}$, where $\sigma$ is the spin index, $\sigma=\uparrow, \downarrow$, and index $n=1,2$ corresponds to left and right ferromagnetic electrodes, respectively. The ferroelectric layer is assumed to be uniformly polarized in the direction perpendicular to the plane. The overall potential in our model is the sum of the rectangular potential which determines the bottoms of the bands and the spin-dependent potential $\phi^{\sigma}(z)$ caused by the spontaneous polarization of the ferroelectric barrier. The latter is obtained by applying shortcircuit boundary conditions and the Thomas-Fermi model of screening taking into account spin-dependent screening [31], as was described previously [43]. The resulting potential can be represented as follows:

$$
\phi^{\sigma}(z)=\frac{\sigma_{\mathrm{S}}}{\varepsilon_{0}} \begin{cases}\lambda_{1}\left(1-\gamma_{1}\right) \mathrm{e}^{z / \lambda_{1}}, & z<0 \\ -\lambda_{2}-\left(\lambda_{1}+\lambda_{2}\right)(z-d) / d, & 0<z<d \\ -\lambda_{2}\left(1-\gamma_{2}\right) \mathrm{e}^{-(z-d) / \lambda_{2}}, & z>d,\end{cases}
$$

where $\sigma_{\mathrm{S}}$ is the screening charge

$$
\sigma_{\mathrm{S}}=\frac{d P}{d+\varepsilon_{\mathrm{f}}\left(\lambda_{1}+\lambda_{2}\right)}
$$

$\varepsilon_{\mathrm{f}}$ is the dielectric constant of ferroelectric at saturation, $\lambda_{n}$ is the screening length in electrode $n(n=1,2)$

$$
\lambda_{n}=\left(\frac{e^{2}}{\varepsilon_{0}} \frac{\rho_{n}+4 J_{n} \rho_{n}^{\downarrow} \rho_{n}^{\uparrow}}{1+J_{n} \rho_{n}}\right)^{-1 / 2},
$$

$\rho_{n}^{\sigma}$ is the spin-dependent density of states at the Fermi energy, $\rho_{n}=\rho_{n}^{\uparrow}+\rho_{n}^{\downarrow}, J_{n}$ is the Stoner exchange parameter, and

$$
\gamma_{n}=\frac{J_{n}\left(\rho_{n}^{\uparrow}-\rho_{n}^{\downarrow}\right)}{1+J_{n} \rho_{n}} .
$$

Figure 1 displays schematically the resulting potential profile of the system.

The IEC energy per unit area is given by $E_{\mathrm{IEC}}=-J \cos \theta$, where $\theta$ is the angle between magnetizations of the two ferromagnetic layers. Positive values of the coupling constant $J$ favor parallel alignment of the magnetizations, and negative values favor antiparallel alignment. To calculate the IEC constant $J$ we use the approach based on the torque produced by rotation of the magnetization of one ferromagnet relative to that of the other $[44,45]$. The torque is related to spin current $j_{\mathrm{s}}$ in a way that allows us to obtain the IEC constant $J$ from the relationship $J \sin \theta=-1 / 2 \hbar j_{\mathrm{s}}$. Assuming that the $z$-axis lies perpendicular to the planes (figure 1), the spin current can be written as follows: $j_{\mathrm{s}}=\operatorname{Re}\left\langle\psi^{\dagger} \sigma_{z} v_{z} \psi\right\rangle$, where $\psi$ is the wavefunction of the system, $v_{z}$ is the $z$ component of the velocity operator $\mathbf{v}=-\mathrm{i} \hbar \nabla / m$, and the angular brackets denote averaging over orbital and spin states which involves integration over the transverse momenta 


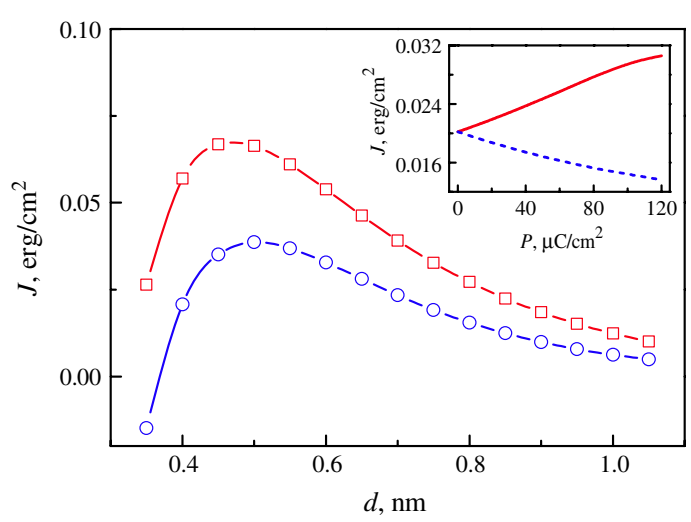

Figure 2. IEC constant $J$ as a function of barrier thickness $d$ for ferroelectric polarization $P$ pointing to the left (squares) and to the right (circles) for $P=75 \mu \mathrm{C} \mathrm{cm}^{-2}, \Delta_{1}=\Delta_{2}=3.6 \mathrm{eV}$, $E_{\mathrm{F}}=2.6 \mathrm{eV}, V_{1}=0$, and $V_{2}=0.6 \mathrm{eV}$. The inset shows $J$ versus $P$ for polarization pointing to the left (dashed line) and to the right (solid line) for $d=0.8 \mathrm{~nm}$

$\mathbf{k}_{\|}$and the energy $E$ weighted with the Fermi distribution function and the summation over contributions from majorityand minority-spin electrons incident from the left and right ferromagnetic layers. The solution of the Schrödinger equation for wavefunction $\psi$ is obtained numerically using appropriate boundary conditions.

Below we investigate the dependence of the IEC constant $J$ on ferroelectric barrier thickness $d$ and band parameters characterizing ferromagnetic electrodes. In all the calculations we set the Fermi energy $E_{\mathrm{F}}=2.6 \mathrm{eV}$ (with respect to the potential energy $V_{1}=0$ ) and fix the exchange splitting in the left electrode $\Delta_{1}=3.6 \mathrm{eV}$. These values may be considered as representative for a Fe electrode [38]. The ferroelectric barrier is described by a free-electron band lying at $U=0.5 \mathrm{eV}$ above the Fermi energy and the effective electron mass $m=0.18 m_{0}$, where $m_{0}$ is a free-electron mass. These values provide the attenuation constant in the barrier consistent with the firstprinciples calculation for a $\mathrm{Pt} / \mathrm{BaTiO}_{3} / \mathrm{Pt}$ ferroelectric tunnel junction (FTJ) [46].

Figure 2 shows the calculated IEC constant $J$ as a function of barrier thickness $d$ for two opposite directions of ferroelectric polarization $P$, the magnitude of which is set to $P=75 \mu \mathrm{C} \mathrm{cm}^{-2}$, appropriate, for example, for $\mathrm{PbTiO}_{3}$. In the calculation we assume the same exchange splitting in the ferromagnetic electrodes $\Delta_{1}=\Delta_{2}$ but different potential energies, i.e. $V_{1}=0, V_{2}=0.6 \mathrm{eV}$. As is seen from figure 2 , there is a pronounced difference between the IEC for polarizations pointing in opposite directions. The IEC is higher for polarization pointing to the right, i.e. in the direction of the electrode with the lower Fermi energy, due to the lower average barrier height for this direction of polarization. The latter behavior is similar to that found in an asymmetric FTJ and is one of the origins of the tunneling electroresistance effect [42]. The difference in the IEC constant for opposite polarization orientations depends on the magnitude of ferroelectric polarization and increases with $P$ almost linearly (see the inset in figure 2). At large barrier thicknesses the IEC coupling decreases exponentially,
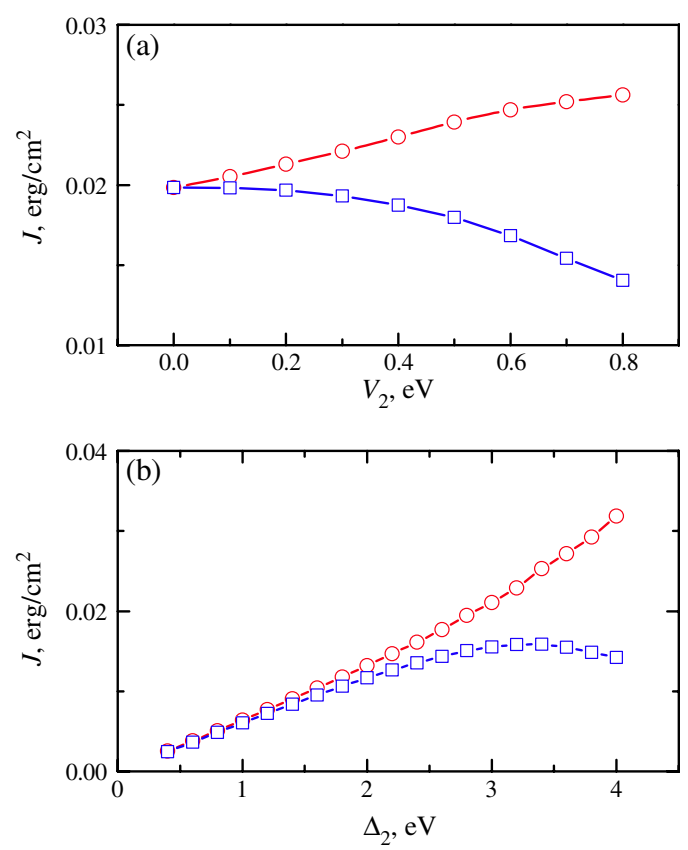

Figure 3. IEC constant $J$ as a function of $V_{2}$ (a) and $\Delta_{2}$ (b) for ferroelectric polarization pointing to the left (squares) and to the right (circles) for $P=75 \mu \mathrm{C} \mathrm{cm}^{-2}, \Delta_{1}=3.6 \mathrm{eV}, E_{\mathrm{F}}=2.6 \mathrm{eV}, V_{1}=0$, and $d=0.8 \mathrm{~nm}$. It is assumed that $\Delta_{2}=3.6 \mathrm{eV}$ (a) and $V_{2}=0.6 \mathrm{eV}(\mathrm{b})$.

as expected from the exponential decay of the wavefunction transmitted through the barrier [44]. The asymmetry in the IEC with respect to polarization orientation is preserved even at large barrier thicknesses. At small thicknesses the coupling reaches a maximum then drops, changing its sign, which is the feature of the IEC calculated within a free-electron model [35]. We note, however, that though the ferroelectricity persists down to vanishingly small thickness of a ferroelectric film, the film must be at least one unit cell thick and hence the minimal value of $d$ is about $0.4 \mathrm{~nm}$.

The change in IEC with ferroelectric polarization reversal is sensitive to asymmetry in the electronic band structure of the two ferromagnetic layers. Figure 3(a) shows the dependence of IEC on $V_{2}$ that determines the band offset between the ferromagnets. In the calculation the exchange splitting of the spin bands in the two ferromagnets was assumed to be the same, i.e. $\Delta_{1}=\Delta_{2}=0.6 \mathrm{eV}$, and barrier thickness was set to be $d=0.8 \mathrm{~nm}$. It seen that the increasing asymmetry in the band structure of the system leads to the greater difference between the IEC constants for opposite ferroelectric polarization orientations. For $V_{2}=0.8 \mathrm{eV}$ the left electrode becomes a half metal that results in a factor of two difference in $J$.

A qualitatively similar effect occurs when the asymmetry in the band structure between the two ferromagnets is introduced through the exchange splitting. This is evident from figure 3(b) which shows the dependence of the IEC constant on $\Delta_{2}$ for different polarization directions. It is seen that the increasing exchange splitting $\Delta_{2}$ in the right electrode (with $\Delta_{1}$ being fixed) results in a significant increase of both $J$ itself and the change in $J$ under polarization switching. 
In conclusion, we have predicted the possibility of IEC between two ferromagnets separated by an ultrathin ferroelectric layer that allows the control of this coupling through ferroelectric polarization orientation. We found that the relative change in the IEC constant depends on the asymmetry in the electronic band structure of the two ferromagnets, and for typical values of ferroelectric polarization it can be as large as a factor of two. The magnitude of the IEC constant has sizable values for subnanometer-thick ferroelectric films but decays exponentially with larger thicknesses of the ferroelectric layer. The predicted phenomenon reveals a new magnetoelectric effect and indicates the possibility of switching the magnetic configuration by reversing the electric polarization of the ferroelectric barrier layer.

This work was supported by the Nebraska Materials Research Science and Engineering Center (MRSEC) (NSF grant no. DMR-0820521), the Nanoelectronics Research Initiative of the Semiconductor Research Corporation, and the Nebraska Research Initiative. AVV is grateful to the Russian Foundation for Basic Research for support.

\section{References}

[1] Schmid H 1994 Ferroelectrics 162665

[2] Fiebig M 2005 J. Phys. D: Appl. Phys. 38 R123

[3] Eerenstein W, Mathur N D and Scott J F 2006 Nature 442759

[4] Ramesh R and Spaldin N A 2007 Nat. Mater. 621

[5] Velev J P, Dowben P A, Tsymbal E Y, Jenkins S J and Caruso A N 2008 Surf. Sci. Rep. 63400

[6] Wang K F, Liu J-M and Ren Z F 2009 Adv. Phys. 58321

[7] Burton J D and Tsymbal E Y 2009 Phys. Rev. B 80174406

[8] Vaz C A F, Hoffman J, Segal Y, Reiner J W, Grober R D, Zhang Z, Ahn C H and Walker F J 2010 Phys. Rev. Lett. 104127202

[9] Borisov P, Hochstrat A, Chen X, Kleemann W and Binek C 2005 Phys. Rev. Lett. 94117203

[10] Laukhin V et al 2006 Phys. Rev. Lett. 97227201

[11] Chu Y-H et al 2008 Nat. Mater. 7478

[12] He X, Wang Y, Wu N, Caruso A N, Vescovo E, Belashchenko K D, Dowben P A and Binek Ch 2010 Nat. Mater 9579

[13] Zhuravlev M Y, Jaswal S S, Tsymbal E Y and Sabirianov R F 2005 Appl. Phys. Lett. 87222114

[14] Tsymbal E Y and Kohlstedt H 2006 Science 313181

[15] Gajek M, Bibes M, Fusil S, Bouzehouane K, Fontcuberta J, Barthélémy A and Fert A 2007 Nat. Mater. 6296

[16] Velev J P, Duan C-G, Burton J D, Smogunov A, Niranjan M K, Tosatti E, Jaswal S S and Tsymbal E Y 2009 Nano Lett. 9427
[17] Garcia V et al 2010 Science 3271106

[18] Duan C-G, Velev J P, Sabirianov R F, Zhu Z, Chu J, Jaswal S S and Tsymbal E Y 2008 Phys. Rev. Lett. 101137201

[19] Weisheit M, Fähler S, Marty A, Souche Y, Poinsignon C and Givord D 2007 Science $\mathbf{3 1 5} 349$

[20] Duan C-G, Velev J P, Sabirianov R F, Mei W N, Jaswal S S and Tsymbal E Y 2008 Appl. Phys. Lett. 92122905

[21] Chiba D, Sawicki M, Nishitani Y, Nakatani Y, Matsukura F and Ohno H 2008 Nature 455515

[22] Maruyama T et al 2009 Nat. Nanotechnol. 4158

[23] Nakamura K, Shimabukuro R, Fujiwara Y, Akiyama T, Ito T and Freeman A J 2009 Phys. Rev. B 80172402

[24] Tsujikawa M and Oda T 2009 Phys. Rev. Lett. 102247203

[25] Dzyaloshinskii I E 1960 Sov. Phys._JETP 10628

[26] Zheng H et al 2004 Science $\mathbf{3 0 3} 661$

[27] Sahoo S, Polisetty S, Duan C-G, Jaswal S S, Tsymbal E Y and Binek C 2007 Phys. Rev. B 76092108

[28] Duan C-G, Jaswal S S and Tsymbal E Y 2006 Phys. Rev. Lett. 97047201

[29] Niranjan M K, Velev J P, Duan C-G, Jaswal S S and Tsymbal E Y 2008 Phys. Rev. B 78104405

[30] Rondinelli J M, Stengel M and Spaldin N 2008 Nat. Nanotechnol. 346

[31] Zhang S 1999 Phys. Rev. Lett. 83640

[32] Niranjan M K, Burton J D, Velev J P, Jaswal S S and Tsymbal E Y 2009 Appl. Phys. Lett. 95052501

[33] Sun Y, Burton J D and Tsymbal E Y 2010 Phys. Rev. B 81064413

[34] Stiles M D 1993 Phys. Rev. B 487238

[35] Bruno P 1995 Phys. Rev. B 52411

[36] Tsymbal E Y, Mryasov O N and LeClair P R 2003 J. Phys.: Condens. Matter 15 R109

[37] Tsymbal E Y, Belashchenko K D, Velev J, Jaswal S S, van Schilfgaarde M, Oleynik I I and Stewart D A 2007 Prog. Mater. Sci. 52401

[38] Zhuravlev M Ye, Tsymbal E Y and Vedyayev A V 2005 Phys. Rev. Lett. 94026806

[39] Tsymbal E Y and Pettifor D G 1999 J. Appl. Phys. 855801

[40] Faure-Vincent J, Tiusan C, Bellouard C, Popova E, Hehn M, Montaigne F and Schuhl A 2002 Phys. Rev. Lett. 89107206

[41] Katayama T, Yuasa S, Velev J, Zhuravlev M Ye, Jaswal S S and Tsymbal E Y 2006 Appl. Phys. Lett. 89112503

[42] Zhuravlev M Ye, Sabirianov R F, Jaswal S S and Tsymbal E Y 2005 Phys. Rev. Lett. 94246802

Zhuravlev M Ye, Sabirianov R F, Jaswal S S and Tsymbal E Y 2009 Phys. Rev. Lett. 102169901

[43] Zhuravlev M Ye, Maekawa S and Tsymbal E Y 2010 Phys. Rev. B 81104419

[44] Slonczewski J C 1989 Phys. Rev. B 396995

[45] Erickson R P, Hathaway K B and Cullen J R 1993 Phys. Rev. B 472626

[46] Velev J P, Duan C-G, Belashchenko K D, Jaswal S S and Tsymbal E Y 2007 Phys. Rev. Lett. 98137201 